\title{
Inclusion in limited distribution drug network reduces time to dalfampridine access in patients with multiple sclerosis at a health-system specialty pharmacy
}

Megan E Peter, PhD; Brandon Markley, PharmD, BCPS; Josh DeClercq, MS; Leena Choi, PhD; Gabrielle Givens, PharmD; Autumn D Zuckerman, PharmD, BCPS, AAHIVP, CSP; and Aimee Banks, PharmD, BCPS, MSCS

\section{What is already known about this subject}

- Accessing specialty medications can be challenging and time consuming, particularly those that are part of a manufacturer limited distribution network (LDN).

- Growing evidence has demonstrated the benefits of integrated healthsystems specialty pharmacies on patient care.

\section{What this study adds}

- This study specifically compares rates and times to medication access for patients prescribed dalfampridine before and after a health-system specialty pharmacy was allowed access in the LDN.

- This study showcases not only the benefits of pharmacist integration on therapy access time, but also the potential limitations and shortcomings of an LDN model.

\begin{abstract}
Author affiliations
Megan E Peter, PhD; Brandon Markley, PharmD, BCPS; Autumn D Zuckerman, PharmD, BCPS, AAHIVP, CSP; and Aimee Banks, PharmD, BCPS, MSCS, Vanderbilt Specialty Pharmacy Services, Vanderbilt University Medical Center, Nashville, TN. Josh DeClercq, MS, and Leena Choi, PhD, Department of Biostatistics, Vanderbilt University Medical Center, Nashville, TN. Gabrielle Givens, PharmD, Lipscomb University College of Pharmacy, Nashville, TN.
\end{abstract}

\section{AUTHOR CORRESPONDENCE:}

Megan E Peter, 615.936.7889;

Megan.peter@vumc.org

J Manag Care Spec Pharm. 2021;27(2):256-62

Copyright (C2021, Academy of Managed Care Pharmacy. All rights reserved.

\section{ABSTRACT \\ BACKGROUND: Dalfampridine improves walking speed in patients with multiple sclerosis (MS), but accessing specialty medications such as dalfampridine can be hindered by insurance restrictions, high costs, and limited distribution networks (LDNs) imposed by manufacturers. Some integrated health-systems specialty pharmacies (HSSPs) embed pharmacists in clinics and dispense medications from their internal pharmacies if included within the LDN.}

OBJECTIVE: To assess access to dalfampridine in patients at an HSSP before and after gaining admission to the LDN.

METHODS: This study was conducted at Vanderbilt Specialty Pharmacy (VSP), an integrated HSSP at Vanderbilt University Medical Center (VUMC) with 2 clinical pharmacists embedded in the MS clinic. VSP gained access to the dalfampridine LDN on May 1 , 2018, at which time the embedded pharmacists began to manage the comprehensive therapy initiation process. We performed a retrospective review of adult patients with MS who were prescribed dalfampridine from March 2010 to December 2018. Eligible prescriptions were new starts (no previous use) or restarts (after previous use and discontinuation). Prescriptions were classified as pre-VSP and post-VSP, which differentiates before and after VSP gained access to dispense dalfampridine. Study outcomes were insurance approval, initiation of therapy, and time from treatment decision to medication access. We used a proportional odds logistic regression model for time to medication 
access using the following covariates: pre-VSP versus post-VSP time period, insurance prior authorization (PA) denied versus approved/ not needed, and baseline timed 25-foot walk.

RESULTS: We included 262 patients and 290 prescriptions (260 preVSP and 30 post-VSP). In pre-VSP and post-VSP prescriptions, $97 \%$ were approved by insurance, and $93 \%$ of patients started therapy. Median time to medication access was 22 days (IQR=11-45) for preVSP prescriptions and 1 day (IQR $=0-3)$ for post-VSP prescriptions. In the proportional odds logistic regression model, the odds of having a longer medication access time were significantly higher for pre-VSP prescriptions $(O R=83.219, P<0.001)$ and prescriptions whose $P A$ was initially denied ( $O R=9.50, P<0.001) ; 25$-foot walk time was not significant $(\mathrm{OR}=0.95, P=0.277)$.

CONCLUSIONS: After obtaining access to dispense dalfampridine, the time to access therapy was reduced, suggesting that LDNs delay patient access to therapy at HSSPs.

Multiple sclerosis (MS) is an immune-mediated disease of the central nervous system that often leads to worsening neurological symptoms and disability. Moreover, gait is often affected in MS patients, which leads to poor balance, higher risk of falls, and the need for a cane or walker., Disease-modifying therapies (DMTs) delay disease progression and prolong time to disability, but patients often require additional treatment to control related symptoms. ${ }^{3,4}$ In addition to nonpharmacologic treatment, the use of the medication dalfampridine may be considered.,5 Approved by the U.S. Food and Drug Administration in 2010, dalfampridine is the only medication indicated to improve walking speed in patients with MS. ${ }^{7}$

However, accessing specialty medications such as dalfampridine can be challenging. Defined as high-cost medications that treat complex, chronic, and/or rare diseases, specialty medications often have specific storage, handling, and administration requirements and require ongoing safety and laboratory monitoring. ${ }^{8}$ Specialty medications also typically require a prior authorization (PA) or appeal for insurance approval and subsequent financial assistance, so patients can afford the medication out-ofpocket cost. ${ }^{9}$ This complex process involves coordination among physicians or other designated clinic staff, insurance company, pharmacy benefit manager, specialty pharmacy, manufacturer, and patient. ${ }^{9}$

An increasing number of health systems are developing integrated health-systems specialty pharmacies (HSSPs), positioning pharmacists within clinics as key members of the health care team to improve medication access, affordability, and monitoring. Growing evidence demonstrates the benefits of this model with high medication access and adherence rates for specialty medications across a range of disease states (e.g., neurology, hematology, and lipid therapy)..$^{10-13}$ However, manufacturers may impose a restriction on medications to be dispensed through a limited distribution network (LDN) of one or a few select pharmacies. ${ }^{14,15}$ This restriction requires patients to obtain specialty medications outside of the integrated HSSP.

Some HSSPs may still assist with the complex insurance approval process to reduce workload on clinic providers if they are unable to ultimately fill the medication; however, this is dependent on bandwidth and resources available at the HSSP. One recent study found that even when HSSPs assist with LDN medication access before transferring the prescription to an in-network pharmacy, time to patient access was still reduced compared with non-LDN medications. ${ }^{12}$ More information is needed to assess the benefits and shortcomings of this distribution model and its effect on patient access to treatment.

The purpose of this study was to compare rates and time to medication access in patients prescribed dalfampridine at an integrated HSSP, before and after the pharmacy gained admission to the LDN.

\section{Methods}

\section{SETTING}

This study was conducted at the outpatient Multiple Sclerosis Clinic at Vanderbilt University Medical Center (VUMC), a large academic health system in the United States. Neurologists in this clinic specialize in treating MS and other central nervous system disorders of the brain and spinal cord. Vanderbilt Specialty Pharmacy (VSP), an integrated pharmacy within VUMC, gained access to dispense dalfampridine through the manufacturer LDN in May 2018.

Before May 1, 2018, dalfampridine prescriptions were managed by nursing staff, who completed paperwork required by insurance providers and submitted paperwork to the drug manufacturer, in addition to their clinical and patient care responsibilities. From May 2018 onward, dalfampridine prescriptions were managed by 2 clinical pharmacists and 2 pharmacy technicians who are embedded in the MS clinic.

Serving as care coordinators for the self-administered MS therapies, the pharmacists provide detailed medication counseling, screen for drug-drug and drug-disease interactions, and ensure that baseline laboratory testing is performed and reviewed. Pharmacists conduct benefits investigations-which include obtaining insurance approval through the PA and appeal if needed-then submit the 
appropriate hub service enrollment form to the manufacturer, ensure that the prescription is triaged to the specialty pharmacy that is within the patient's insurance network (if not filled internally), and help patients enroll in copay assistance. After patients start therapy, pharmacists monitor ongoing adherence and safety labs and manage adverse drug reactions. A previous study conducted in the VUMC MS clinic demonstrated that adherence to DMTs far exceeded the industry standard, indicating the benefits of embedded clinical pharmacists and their ability to minimize clinical, financial, and LDN restrictions..$^{10}$

\section{STUDY DESIGN}

We conducted a single-center retrospective review of adult patients with a diagnosis of MS (International Classification of Diseases, Tenth Revision, Clinical Modification code G35) who were prescribed dalfampridine by a VUMC provider from March 2010 to March 2020. Eligible prescriptions were new therapy starts (no previous dalfampridine use) or restarts (previous dalfampridine initiation and discontinuation at least 3 months before). Prescriptions were excluded if they were initiated with an external pharmacy by a nonVUMC provider or if the original prescription was not found in the electronic health record. Data were extracted from electronic health records and pharmacy claims. Prescriptions were then classified as pre-VSP (generated before May 1, 2018) or post-VSP (generated on or after May 1, 2018). The Vanderbilt University Institutional Review Board approved the study.

\section{MEASURES}

We collected age, gender, and race of eligible patients. For each included prescription, we collected the following: date of decision to treat; treatment indication; whether an insurance PA and appeal was needed, and if so, PA/appeal approved or denied along with the subsequent date of approval or denial; and whether the patient started medication. When available, we collected patient ambulatory status, Expanded Disability Status Scale (EDSS) score, and timed 25-foot walk (T25FW) conducted within 3 months before decision to treat. Study data were stored in REDCap, which is hosted at Vanderbilt University. ${ }^{16}$

\section{OUTCOMES}

The primary outcome was time to medication access, defined as the number of days between treatment decision date and insurance approval. Secondary outcomes were the percentage of prescriptions approved by insurance and the percentage of patients who started prescribed therapy.

\section{STATISTICAL ANALYSIS}

Categorical variables were described using frequency distributions, and continuous variables were summarized using means, SDs, medians, and interquartile ranges (IQR).

To account for patients with missing data in our regression analysis, rather than deleting them, we performed multiple imputations. Using the aregImpute function from the R package rms, version 6.1-0 (Frank E. Harrell, Jr., Department of Biostatistics, Vanderbilt University, Nashville, TN), we created 20 imputation datasets using the predictive mean matching method. Variables included in the imputation were age, weight, diagnosis, insurance, T25FW, patient status at time of data collection, serum creatinine, estimated glomerular filtration rate, whether the patient was on DMT, EDSS score, ambulatory status, financial assistance, manufacturer hub support services utilization, and VSP utilization, as well as multiple variables related to the approval process. Using the imputed data, we fit a proportional odds logistic regression model for time to medication access using the following covariates: time period (pre-VSP vs. post-VSP), PA outcome (denied vs. approved/not needed), and T25FW in seconds. Parameter estimates were averaged over the 20 imputed datasets. Data were analyzed using the programming language R version 4.0.2 (R Foundation for Statistical Computing, Vienna, Austria).

\section{Results}

We included 262 patients: 68\% women and $88 \%$ Caucasian, with a median age of 52 years $(\mathrm{IQR}=45-60)$. Twenty-seven patients had multiple dalfampridine prescriptions during the study period (233 patients with $\geq 1$ prescriptions pre-VSP, 20 with $\geq 1$ prescriptions post-VSP, and 9 with $\geq 1$ prescriptions in pre-VSP and post-VSP), resulting in 290 prescriptions: 260 pre-VSP and 30 post-VSP. Most common associated diagnoses for prescriptions were relapsing remitting MS (42\%) and secondary progressive MS (37\%). At baseline, T25FW was conducted for $89 \%$ (231) of pre-VSP and $83 \%$ (25) of post-VSP prescriptions. Prescription characteristics are summarized in Table 1.

In pre-VSP and post-VSP prescriptions, 97\% were approved by insurance, and $93 \%$ of patients started therapy. Reasons that patients did not start therapy are summarized in Table 1. Median time to medication access was 22 days $(\mathrm{IQR}=11-45)$ for pre-VSP prescriptions and 1 day $(\mathrm{IQR}=0-3)$ for post-VSP prescriptions. Annual median access times in the 8.5-year pre-VSP time period ranged from 14 to 42 days. 


\section{TABLE 1 Characteristics of Dalfampridine Prescriptions}

\begin{tabular}{|c|c|c|c|c|}
\hline \multirow{3}{*}{ Insurance pathway and outcome } & \multicolumn{2}{|c|}{ Pre-VSPa $(n=260)$} & \multicolumn{2}{|c|}{ Post-VSPb $(n=30)$} \\
\hline & \multicolumn{4}{|c|}{$\%(n)$ or median [IQR] } \\
\hline & & & & \\
\hline PA not applicable (patient uninsured) & $<1$ & $(1)$ & 0 & $(0)$ \\
\hline PA not required $\rightarrow$ insurance approved & 26 & $(68)$ & 20 & (6) \\
\hline PA required, submitted, and approved $\rightarrow$ insurance approved & 67 & $(173)$ & 70 & $(21)$ \\
\hline PA required, submitted, and denied $\rightarrow$ appeals submitted $\rightarrow$ insurance approved & 4 & $(11)$ & 7 & $(2)$ \\
\hline PA required, submitted, and denied $\rightarrow$ appeals submitted $\rightarrow$ insurance not approved & 1 & $(2)$ & 3 & (1) \\
\hline PA required, submitted, and denied $\rightarrow$ no appeal submitted $\rightarrow$ insurance not approved & 2 & $(5)$ & 0 & $(0)$ \\
\hline \multicolumn{5}{|l|}{ Insurance approval outcome } \\
\hline Insurance approved & 97 & $(253)$ & 97 & $(29)$ \\
\hline Insurance did not approve & 3 & (7) & 3 & $(1)$ \\
\hline \multicolumn{5}{|l|}{ Patient started medication } \\
\hline Yes & 93 & $(241)$ & 93 & $(28)$ \\
\hline No & 7 & (19) & 7 & $(2)$ \\
\hline \multicolumn{5}{|l|}{ Reason patient did not start medication $(n=20)$} \\
\hline Loss to follow-up & 16 & (3) & 0 & $(0)$ \\
\hline Patient preference & 21 & (4) & 50 & (1) \\
\hline Rx not approved & 32 & $(5)$ & 50 & (1) \\
\hline Copay too high & 26 & $(5)$ & 0 & $(0)$ \\
\hline High cost of labs & 5 & (1) & 0 & (0) \\
\hline \multicolumn{5}{|l|}{ Diagnosis } \\
\hline Primary progressive multiple sclerosis & 19 & $(49)$ & 33 & $(10)$ \\
\hline Relapse remitting multiple sclerosis & 43 & $(112)$ & 30 & (9) \\
\hline Secondary progressive multiple sclerosis & 37 & (97) & 37 & (11) \\
\hline Transverse myelitis & 1 & (2) & 0 & $(0)$ \\
\hline Data not available & 25 & $(66)$ & 23 & (7) \\
\hline Expanded Disability Status Scale score $(n=100)$ & 6.0 & {$[4.0-6.5]$} & 5.5 & {$[4.0-6.5]$} \\
\hline \multicolumn{5}{|l|}{ Ambulatory $(n=286)$} \\
\hline Yes & 93 & $(238)$ & 97 & $(28)$ \\
\hline No & 7 & (19) & 3 & $(1)$ \\
\hline Baseline timed 25 -foot walk, seconds $(n=256)^{c}$ & 8.9 & {$[6.7-15.3]$} & 11.0 & [6.3-14.8] \\
\hline Access time, days $(n=279)$ & 22.0 & [11.0-45.0] & 1.0 & {$[0.0-3.0]$} \\
\hline
\end{tabular}

In the proportional odds logistic regression model, summarized in Table 2, medication access time was significantly longer for pre-VSP prescriptions (odds ratio
$[\mathrm{OR}]=83.219, \mathrm{P}<0.001)$ and prescriptions for which $\mathrm{PA}$ was initially denied $(\mathrm{OR}=9.50, \mathrm{P}<0.001)$; T25FW was not significant $(\mathrm{OR}=0.95, \mathrm{P}=0.277)$. 


\section{TABLE 2 Proportional Odds Logistic Regression Predicting Time to Medication Access}

\begin{tabular}{l|c|c|c|c}
\hline Characteristic & Odds Ratio & Lower CI & Upper CI & P Value \\
\hline Pre-VSP vs. post-VSP & 83.20 & 32.80 & 21.10 & $<0.001$ \\
\hline PA denied vs. PA approved/not needed & 9.50 & 4.30 & 21.10 & $<0.001$ \\
\hline Timed 25-foot walk score (per 5 seconds) & 0.95 & 0.88 & 1.04 & 0.280 \\
\hline
\end{tabular}

Note: Using the imputed data, we fit a proportional odds logistic regression model for time to medication access. Parameter estimates were averaged over the 20 imputed datasets.

$\mathrm{Cl}=$ confidence interval; $\mathrm{PA}=$ prior authorization; $\mathrm{VSP}=$ Vanderbilt Specialty Pharmacy.

\section{Discussion}

The purpose of this study was to determine rates and timing of medication access for patients who were prescribed dalfampridine at an outpatient MS clinic with integrated specialty pharmacy services before and after access to the manufacturer LDN. Treatment delays, decreased medication adherence, and general increased inefficiencies in the medication use process may lead to worse patient clinical outcomes. ${ }^{17-21}$

Obtaining patient access to specialty medications can be time consuming and burdensome for prescribers and clinic staff, requiring communication between clinics, insurance providers, drug manufacturers, pharmacy benefit managers, specialty pharmacies, and patients, on top of the clinician's day-to-day patient care duties. ${ }^{9}$ We compared outcomes during 2 time periods: preVSP, when VSP did not have access to the LDN for dalfampridine, and postVSP, when the LDN restriction was removed and the medication could be dispensed from VSP, allowing the clinic-embedded pharmacy staff to manage the therapy initiation process.

We found that rates of insurance approval were similar between the 2 time periods, but time to medication access-defined as the number of days between decision to treat and insurance approval-decreased from a median of 22 days to 1 day after the LDN restriction was removed.

Although we did not assess whether speed of medication access affected patient clinical outcomes, we found that patients and providers are more satisfied when medication can be accessed quickly and efficiently, and faster access allows patients to glean benefits of medication sooner. These findings contribute to emerging evidence exploring how LDNs may prevent or delay patients from accessing necessary therapy.

With multiple logistic regression, we explored whether PA approval and T25FW were associated with access time, after accounting for the difference between time periods. Results indicated that access time was significantly longer for prescriptions in the pre-VSP time period and prescriptions whose PA was denied. We hypothesized that patients' baseline T25FW could be associated with how quickly insurance providers approved medication, since payers may use this test to help predict whether dalfampridine would be beneficial for a patient; however, patients' baseline T25FW (collected within 3 months before prescription generation) were not associated with access time.

Removing the LDN restriction allowed streamlined and faster patient access to medication, yet regardless of whether drugs were limited distribution, barriers obtaining insurance coverage delayed time to medication access. This is not surprising, since obtaining medication approval and financial assistance coverage is often complex and time consuming. One study of oncology medications listed 17 steps required for medication approval, and another reported that clinic staff spent a median of 2 hours per prescription on medication access requirements. ${ }^{22,23}$

Although oncology medications may entail different insurance requirements than for MS medications, both are high-cost specialty therapies that require pharmacy benefit managers to ensure that they are safe and appropriate for a patient before approving; these requirements demand time and resources from clinic staff to ensure that patients initiate therapy. Our study results suggest that integrated HSSPs can reduce clinic workload and streamline patient access to therapy.

Removing the LDN restriction allowed our clinic and pharmacy to better coordinate patient access to therapy. Before VSP received access to dispense dalfampridine, prescriptions were managed by clinic nursing staff, who had to follow a cumbersome process. The prescription/start form was submitted to the manufacturer hub to process and determine if the patient's insurance required a PA; if so, the clinic nursing staff were notified to complete a PA. After the PA was approved, a prescription was sent to an external specialty pharmacy that dispensed the medication. This approach to therapy initiation may lead to delays in treatment because of the need for frequent, back and forth communication between the clinic staff and manufacturer hub.

After the LDN restriction was removed, the embedded clinic pharmacy staff began managing dalfampridine prescriptions. Prescribers notified the pharmacists when the decision was made 
to initiate dalfampridine. The pharmacist then reviewed the patient's electronic medical record for medication appropriateness (including drug-drug interactions, drug-disease interactions, and necessary baseline laboratory testing) and provided detailed medication counseling to the patient. An insurance benefits investigation was completed (to determine the copay amount, deductible, coverage gaps and limits, if a PA is needed, and if a particular specialty pharmacy is required for dispensing); the pharmacist completed the PA and appeal, if necessary, until insurance approved the medication.

Following insurance approval, patient financial needs were reviewed and additional assistance was pursued, if necessary. Thus, once the prescription was received by the appropriate specialty pharmacy, whether VSP or an external pharmacy, the medication could be immediately shipped to the patient.

Pharmacists, as medication experts, are best suited to manage clinical, financial, and administrative aspects of specialty medication prescriptions, and this allows clinic nurses to better focus their time and attention on direct patient care in the clinic. Although not assessed in this study, we hypothesized that after inclusion into the LDN network, insurance requirements were completed more quickly than in the pre-VSP time frame.

Additionally, pharmacists might complete more thorough PAs because they are familiar with specific insurance requirements and common reasons for insurance denial, reducing the need to seek clinical information from providers for clarification before submission. Future research is warranted to assess hours saved and thus costs saved by the health system after devoting a pharmacist and pharmacy technician to managing these tasks.
Developed with patient safety in mind, LDNs ensure that one or few select specialty pharmacies conduct thorough medication counseling and safety monitoring for patients prescribed high-cost or high-risk specialty drugs. They also may secure product integrity and enable manufacturers to capture more data and generate more revenue. . $^{14,24}$

Conversely, health systems have voiced concerns regarding the negative effect of LDNs on patients, providers, and health systems. ${ }^{14,15,24,25}$ LDNs introduce a potential to fragment care and, as demonstrated by our results, may prolong time to medication access for patients. Pharmacists at integrated HSSPs are well positioned to coordinate care, streamline medication access, and facilitate communication among clinic providers, manufacturers, insurers, and patients.

Additionally, integrated HSSPs monitor patients after therapy is initiated-through refill calls, pharmacist assessments, and clinic visits-to ensure ongoing safety, effectiveness, and appropriateness of therapy. When included in an LDN, the comprehensive services offered by HSSPs can ensure that patients receive cohesive care.

\section{LIMITATIONS}

This study has limitations to consider. The sample size is small, notably for post-VSP prescriptions, and data were collected from a single-center MS clinic in the southeastern United States. Thus, findings may not generalize to all patient populations.

The pre-post design limits the ability to draw causal conclusions on the effect of integrated specialty pharmacies on medication access time; however, patient and prescription characteristics were similar between groups.

Also, the pre-VSP time frame was much longer than the post-VSP period, and changes within the clinic or payer formularies or requirements could contribute to fluctuations over time; however, access time remained relatively stable during the pre-VSP time period. Studies are needed to demonstrate the clinical effect of reducing time to dalfampridine treatment on patient outcomes.

\section{Conclusions}

After the integrated specialty pharmacy obtained access to dispense dalfampridine, patients accessed therapy more quickly, suggesting that LDNs delay access to specialty medications for patients of health systems with integrated specialty pharmacies. When LDNs are removed, our integrated specialty pharmacists can provide medication monitoring, counseling, and safety interventions after patients initiate treatment to promote high adherence and persistence to therapy.

\section{DISCLOSURES}

No funding was provided for this study. The authors have no conflicting interests to disclose.

Preliminary results have been previously presented at the American Society of Health-Systems Pharmacy Midyear Meeting in December 2019, the Vanderbilt Health Systems Specialty Pharmacy Outcomes Research Summit in August 2020, and the National Association of Specialty Pharmacy Annual Meeting in September 2020.

\section{REFERENCES}

1. Compston A, Coles A. Multiple sclerosis. Lancet. 2008;372(9648):1502-17.

2. Frohman TC, Castro W, Shah A, et al. Symptomatic therapy in multiple sclerosis. Ther Adv Neurol Disord. 2011;4(2):83-98. 
3. Goodin DS, Frohman EM, Garmany GP Jr, et al. Disease modifying therapies in multiple sclerosis: report of the Therapeutics and Technology Assessment Subcommittee of the American Academy of Neurology and the MS Council for Clinical Practice Guidelines. Neurology. 2002;58(2):169-78.

4. Rae-Grant A, Day GS, Marrie RA, et al. Practice guideline recommendations summary: disease-modifying therapies for adults with multiple sclerosis: report of the Guideline Development, Dissemination, and Implementation Subcommittee of the American Academy of Neurology. Neurology. 2018;90(17):777-88.

5. Brownlee WJ, Hardy TA, Fazekas F, Miller DH. Diagnosis of multiple sclerosis: progress and challenges. Lancet. 2017;389(10076):1336-46.

6. Reich DS, Lucchinetti CF, Calabresi PA. Multiple sclerosis. N Engl J Med. 2018;378(2):169-80.

7. AMPYRA (dalfampridine) extended release tablets, for oral use. Acorda Therapeutics. Revised January 2013. Accessed December 31, 2020. https:// www.accessdata.fda.gov/drugsatfda_ docs/label/2013/022250s006lbl.pdf

8. National Association of Specialty Pharmacy. NASP Definitions of specialty pharmacy and specialty medications. February 2016. Accessed December 31, 2020. http://naspnet.org/wp-content/ uploads/2017/02/NASP-Defintionsfinal-2.16.pdf

9. Cocohoba J, Pohlman B, Tran JS, et al. Modeling specialty medicine access: understanding key health system processes and players. J Am Pharm Assoc (2003). 2019;59(1):43-50.e43.
10. Banks AM, Peter ME, Holder GM, et al. Adherence to disease-modifying therapies at a multiple sclerosis clinic: the role of the specialty pharmacist. J Pharm Pract. 2020;33(5):605-11.

11. Reynolds VW, Chinn ME, Jolly JA, et al. Integrated specialty pharmacy yields high PCSK9 inhibitor access and initiation rates. J Clin Lipidol. 2019;13(2):254-64.

12. Wyatt H, Peter M, Zuckerman A, et al. Assessing the impact of limited distribution drug networks based on time to accessing oral oncolytic agents at an integrated specialty pharmacy. J Hematol Oncol Pharm. 2020;10(4):198-205.

13. Berger N, Peter M, DeClercq J, Choi L, Zuckerman A. Rheumatoid arthritis medication adherence in a health-system specialty pharmacy. Am J Manag Care. 2020;26(12):e380-e387.

14. Karas L, Shermock KM, Proctor C, Socal M, Anderson GF. Limited distribution networks stifle competition in the generic and biosimilar drug industries. Am J Manag Care. 2018;24(4):e122-e127.

15. Kracov DA. Legal issues and impact of restricted access and distribution. J Am Pharm Assoc (Wash). 2001;41 (6 Suppl 1):S11-S15.

16. Harris PA, Taylor R, Thielke R, Payne J, Gonzalez N, Conde JG. Research electronic data capture (REDCap)-a metadata-driven methodology and workflow process for providing translational research informatics support. J Biomed Inform. 2009;42(2):377-81.
17. Devonshire V, Lapierre Y, Macdonell R, et al. The Global Adherence Project (GAP): a multicenter observational study on adherence to disease-modifying therapies in patients with relapsingremitting multiple sclerosis. Eur J Neurol. 2011;18(1):69-77.

18. Higuera L, Carlin CS, Anderson S. Adherence to disease-modifying therapies for multiple sclerosis. J Manag Care Spec Pharm. 2016;22(12):1394-401. doi: 10.18553/ jmcp.2016.22.12.1394

19. Patti F. Optimizing the benefit of multiple sclerosis therapy: the importance of treatment adherence. Patient Prefer Adherence. 2010;4:1-9.

20. Schultz J, Weber C, Kamholz J. Letter to the editor: the emerging role of pharmacists in the multidisciplinary care of patients with multiple sclerosis. Int J MS Care. 2016;18(5):219-20.

21. Tobin WO, Weinshenker BG. Stopping immunomodulatory medications in MS: frequency, reasons and consequences. Mult Scler Relat Disord. 2015;4(5):437-43.

22. Agarwal A, Freedman RA, Goicuria F, et al. Prior authorization for medications in a breast oncology practice: navigation of a complex process. J Oncol Pract. 2017;13(4):e273-e282.

23. Niccolai JL, Roman DL, Julius JM, Nadour RW. Potential obstacles in the acquisition of oral anticancer medications. J Oncol Pract. 2017;13(1):e29-e36.

24. Gabay MP, Costianis R. Restricted drug distribution programs. Am J Health Syst Pharm. 2003;60(15):1525-30.

25. Turingan EM, Mekoba BC, Eberwein SM, et al. Financial effect of a drug distribution model change on a health system. Hosp Pharm. 2017;52(6):422-27. 